\title{
Effect of proximal translation of the osteotomized tibial tuberosity during tibial tuberosity advancement on patellar position and patellar ligament angle
}

\author{
Jack D. Neville-Towle*, Mariano Makara, Kenneth A. Johnson and Katja Voss
}

\begin{abstract}
Background: Cranial cruciate ligament insufficiency is a common orthopaedic problem in canine patients. This cadaveric and radiographic study was performed with the aim of determining the effect of proximal translation of the tibial tuberosity during tibial tuberosity advancement (TTA) on patellar position (PP) and patellar ligament angle (PLA).

Results: Disarticulated left hind limb specimens harvested from medium to large breed canine cadavers $(n=6)$ were used for this study. Limbs were mounted to Plexiglass sheets with the stifle joint fixed in $135^{\circ}$ of extension. The quadriceps mechanism was mimicked using an elastic band. Medio-lateral radiographs were obtained pre-osteotomy, after performing TTA without proximal translation of the tibial tuberosity, and after proximal translation of the tibial tuberosity by $3 \mathrm{~mm}$ and $6 \mathrm{~mm}$. Radiographs were blinded to the observer for distance of tibial tuberosity proximalization following radiograph acquisition. Three independent observers recorded PP and PLA (tibial plateau method and common tangent method). Comparisons were made between the stages of proximalization using repeated measures ANOVA. Patellar position was found to be significantly more distal than pre-osteotomy, if the tibial tuberosity was not translated proximally $(P=0.001)$ and if it was translated proximally by $3 \mathrm{~mm}(P=0.005)$. The difference between pre-osteotomy PP and $6 \mathrm{~mm}$ proximalization was not significant. The PLA was significantly larger if the tibial tuberosity was not translated proximally compared to tibial tuberosity proximalization of $6 \mathrm{~mm}$ using the tibial plateau and the common tangent methods ( $P=0.006$ and $P=0.015$ respectively).
\end{abstract}

Conclusions: Proximalizing the tibial tuberosity during TTA helps in maintaining vertical position of the patella in the patellar groove. Proximalization of the tibial tuberosity reduces PLA when compared to TTA without tibial tuberosity proximalization.

Keywords: Cranial cruciate ligament, Tibial tuberosity advancement, Patellar position, Canine

\section{Background}

Cranial cruciate ligament disease is the most commonly diagnosed cause of osteoarthritis and lameness in the canine stifle [1-4]. Given the prevalence of disease, development of treatment techniques, including various tibial osteotomies have been described over the past 25 years [5-9]. These procedures are intended to change stifle dynamic joint biomechanics rather than directly repair the cranial cruciate ligament and include the cranial

\footnotetext{
* Correspondence: jack.neville-towle@sydney.edu.au University Veterinary Teaching Hospital, Sydney, Faculty of Veterinary Science, University of Sydney, Sydney, NSW 2006, Australia
}

tibial wedge osteotomy [6] the tibial plateau levelling osteotomy [7], the tibial tuberosity advancement [8], and the triple tibial osteotomy [9].

The TTA technique was first described in 2002 and aims to neutralise cranial shear force of the tibia by reducing the patellar ligament angle to $90^{\circ}$ [8]. Reduction of the patellar ligament angle is achieved by osteotomizing and advancing the tibial tuberosity cranially. Clinically, cranial advancement of the tibial tuberosity can be performed either with or without concurrent proximal translation of the distal end of the tibial tuberosity. With the original TTA technique, the advanced tibial tuberosity is stabilized 
using a cage, which prevents collapse of the osteotomy, and a tension band plate, which counteracts the tensile forces exerted on the tibial tuberosity by the quadriceps muscles and the patellar ligament $[8,10]$.

In recent years, several modifications of the TTA technique have been described. One of these modifications is the modified Maquet technique or more recently the modified Maquet procedure (MMP) [5, 11-13]. The original Maquet technique was described for humans in 1976 and involved advancement of tibial tuberosity with the aim of reducing retropatellar pressure in patients with femoropatellar osteoarthritis [13]. Subsequently, the concept of the MMP involved a proximal tibial osteotomy similar to that described by Montavon et al. [8] but instead of using a plate to counteract the tensile forces exerted on the tibial tuberosity by the quadriceps mechanism, these forces are counteracted by performing an incomplete osteotomy that leaves a cortical hinge distally [5]. Collapse of the osteotomy can be prevented by insertion of a TTA cage or another spacer and a number of variations of this procedure have been reported [12, 14, 15]. Initial clinical and biomechanical studies suggest that the technique is biomechanically sound and may be safely applied in clinical patients $[5,11,16]$.

With the original TTA technique, several millimetres of proximal tibial tuberosity translation has been recommended to maintain vertical position of the patella during advancement [10]. The TTA technique can also be used to correct pre-existing patella baja or alta by shifting the tibial tuberosity proximally or distally, respectively [17]. With the MMP, it is not possible to shift the tibial tuberosity proximally as the technique relies on the presence of a distal cortical hinge around which the tibial tuberosity is rotated cranially. Rotation of the tibial tuberosity around the distal cortical hinge could result in patella baja as the insertion of the patellar ligament moves craniodistal to the patella during advancement. Studies reported in the human literature have shown that there is a $15 \%$ decrease in patella height after a similar procedure with $26 \%$ of patients meeting newonset patella baja criteria postoperatively [18, 19].

A recent trigonometric study has shown that there is a discrepancy between preoperative PLA measurements and the actual PLA achieved by advancing the tibial tuberosity as calculated [20]. The findings of this study have since been substantiated clinically with actual tibial tuberosity advancement achieved during the MMP being $30 \%$ lower than the calculated wedge size used [14]. This discrepancy is worse in dogs with a steep tibial plateau and when larger cage sizes are used. The consequence of this is a potential failure to achieve a $90^{\circ}$ PLA postoperatively [20]. Discrepancies have also been noted between the tibial plateau slope and common tangent methods of determining PLA at varying degrees of stifle extension
[21]. This highlights the importance of consistent limb positioning in assuring the accuracy of these measurements [21-25]. The effect of proximalization of the tibial tuberosity during advancement on postoperative PLA is currently unknown.

The aims of this study were to provide an ex vivo comparison of PP and PLA following TTA with varying degrees of proximal translation of the tibial tuberosity $(0 \mathrm{~mm}, 3 \mathrm{~mm}$ and $6 \mathrm{~mm})$. We hypothesized that proximalization of the tibial tuberosity during TTA would have a significant effect on PP and the PLA.

\section{Results}

The breeds of the dogs used for this study were mixedbreed $(n=3)$, Labrador $(n=1)$, Rottweiler $(n=1)$ and Australian Cattle Dog $(n=1)$. Body weight ranged from 23.2 to $41.0 \mathrm{~kg}$ with a mean of $33.0( \pm 6.4 \mathrm{~kg})$. Cage sizes used based on pre-osteotomy measurements were $12 \mathrm{~mm}(n=3), 10.5 \mathrm{~mm}(n=2)$ and $9 \mathrm{~mm}(n=1)$. Radiographic assessment of the $3 \mathrm{~mm}$ and $6 \mathrm{~mm}$ tibial tuberosity proximalizations showed that the $3 \mathrm{~mm}$ stage had a mean proximalization of $3.17( \pm 0.55 \mathrm{~mm})$ and the $6 \mathrm{~mm}$ stage a mean of $5.90( \pm 0.46 \mathrm{~mm})$ respectively.

\section{Patellar position measurements}

Inter-observer agreement for the PP measurements was 0.9 (95\% CI: 0.723-0.960).

Mean pre-osteotomy PP measurement was $28.0 \mathrm{~mm}( \pm$ 0.32 ). This distance was reduced post-osteotomy to $24.3 \mathrm{~mm}( \pm 0.34)$ if the tibial tuberosity was not proximally translated, $25.4 \mathrm{~mm}( \pm 0.29)$ if it was proximally translated by $3 \mathrm{~mm}$, and $26.8 \mathrm{~mm}( \pm 0.33)$ if it was proximally translated by $6 \mathrm{~mm}$ (Fig. 1). Bonferroni pair-wise comparisons indicated that the patella was positioned significantly lower than pre-osteotomy measurements if the tibial tuberosity was not translated proximally $(P=$ $0.001)$ and if it was translated proximally by $3 \mathrm{~mm}(P=$ 0.005). Difference between $3 \mathrm{~mm}$ and $6 \mathrm{~mm}$ proximal translations was also significant $(P=0.002)$ whilst the difference between pre- and postoperative patellar position with the tibial tuberosity proximalized by $6 \mathrm{~mm}$ was not. The difference between $0 \mathrm{~mm}$ and $3 \mathrm{~mm}$ proximal translations was also not significant.

\section{Patellar ligament angle measurements (PLAtangent and PLAslope)}

There was excellent interobserver agreement for both PLA measurements with an agreement of 0.922 (95\% CI: 0.822-0.966) for PLAslope and 0.925 (95\% CI: 0.660.975) for PLAtangent.

Both PLAtangent and PLAslope decreased with increased proximal translation of the tibial tuberosity. Mean preoperative PLAtangent was $98.97^{\circ}( \pm 3.41)$. Mean postoperative PLAtangent was $87.46^{\circ}( \pm 3.47), 86.42^{\circ}( \pm 2.88)$ and $83.85^{\circ}$ 




Fig. 1 Patellar Position Error chart (+/ $1 \mathrm{SE}$ ) representing the change in patella position $(\mathrm{mm})$ between tibial tuberosity proximal translation stages. Statistically significant difference was observed between the pre-osteotomy and 0mm, pre-osteotomy and $3 \mathrm{~mm}$, 0mm and $6 \mathrm{~mm}$ and $3 \mathrm{~mm}$ and $6 \mathrm{~mm}$ stages

$( \pm 3.67)$ at $0 \mathrm{~mm}, 3 \mathrm{~mm}$ and $6 \mathrm{~mm}$ of proximal translation of the tibial tuberosity, respectively (Fig. 2). Mean preoperative PLAslope was $105.78^{\circ}( \pm 2.58)$. Postoperative PLAslopes were $96.92^{\circ}( \pm 3.40), 95.65^{\circ}( \pm 3.26)$ and $94.37^{\circ}( \pm 3.39)$ at $0 \mathrm{~mm}, 3 \mathrm{~mm}$ and $6 \mathrm{~mm}$ of proximal translation (Fig. 3). Differences between groups were significant $(P=0.001$ for PLAtangent; $P<0.001$ for PLAslope). Pair-wise comparisons using Bonferroni adjustment revealed significant differences between PLAslope $(P=0.006)$ and PLA tangent angles $(P=0.015)$ at $0 \mathrm{~mm}$ and $6 \mathrm{~mm}$ of tibial tuberosity proximalization. The differences between $3 \mathrm{~mm}$ and $6 \mathrm{~mm}$ were significantly different for PLAtangent $(P=0.002)$, but not PLAslope. The difference between the $0 \mathrm{~mm}$ and $3 \mathrm{~mm}$ stages was not significant for PLAslope or PLAtangent.

\section{Discussion}

This study has shown that failure to translate the tibial tuberosity proximally during TTA results in significant distal displacement of the patella as compared to shifting the tibial tuberosity proximally by $6 \mathrm{~mm}$. Additionally, postosteotomy PLAs (PLAtangent and PLAslope) were larger when the tibial tuberosity was not proximally translated.

Several methods exist to quantify PP. We chose the Blackburne and Peel [26] method because it has been shown to have less interobserver error when compared to other commonly used techniques [27]. With the specimen in a fixed position between radiographs the common tangent of the femoro-tibial articulation was considered a consistent reference from which to measure [21, 28, 29]. We found excellent interobserver agreement using this method.

Significant distal patella displacement occurred when the tibial tuberosity was not proximalized or only proximalized by $3 \mathrm{~mm}$, compared to pre-osteotomy measurements. This supports the description to proximalize the tibial tuberosity during TTA in order to maintain patellar position [10]. In the group of dogs in this study with a mean weight of $33 \mathrm{~kg}$, the mean distal displacement occurring without translation of the tibial tuberosity as compared to pre-osteotomy was $3.7 \mathrm{~mm}$. The clinical implication of distal patella displacement as shown in this study is unknown. Additionally, a relationship between patella baja and increased incidence of congenital lateral patellar luxation has been described in dogs [30]. From our data, it is not possible to state whether the observed degree of distal patellar displacement could increase the risk for lateral patellar luxation postoperatively. The degree of distal patellar displacement during TTA is likely to be larger when larger cage sizes are used because the patellar ligament insertion will be pulled increasingly more distally with increasing degrees of tibial tuberosity advancement. 


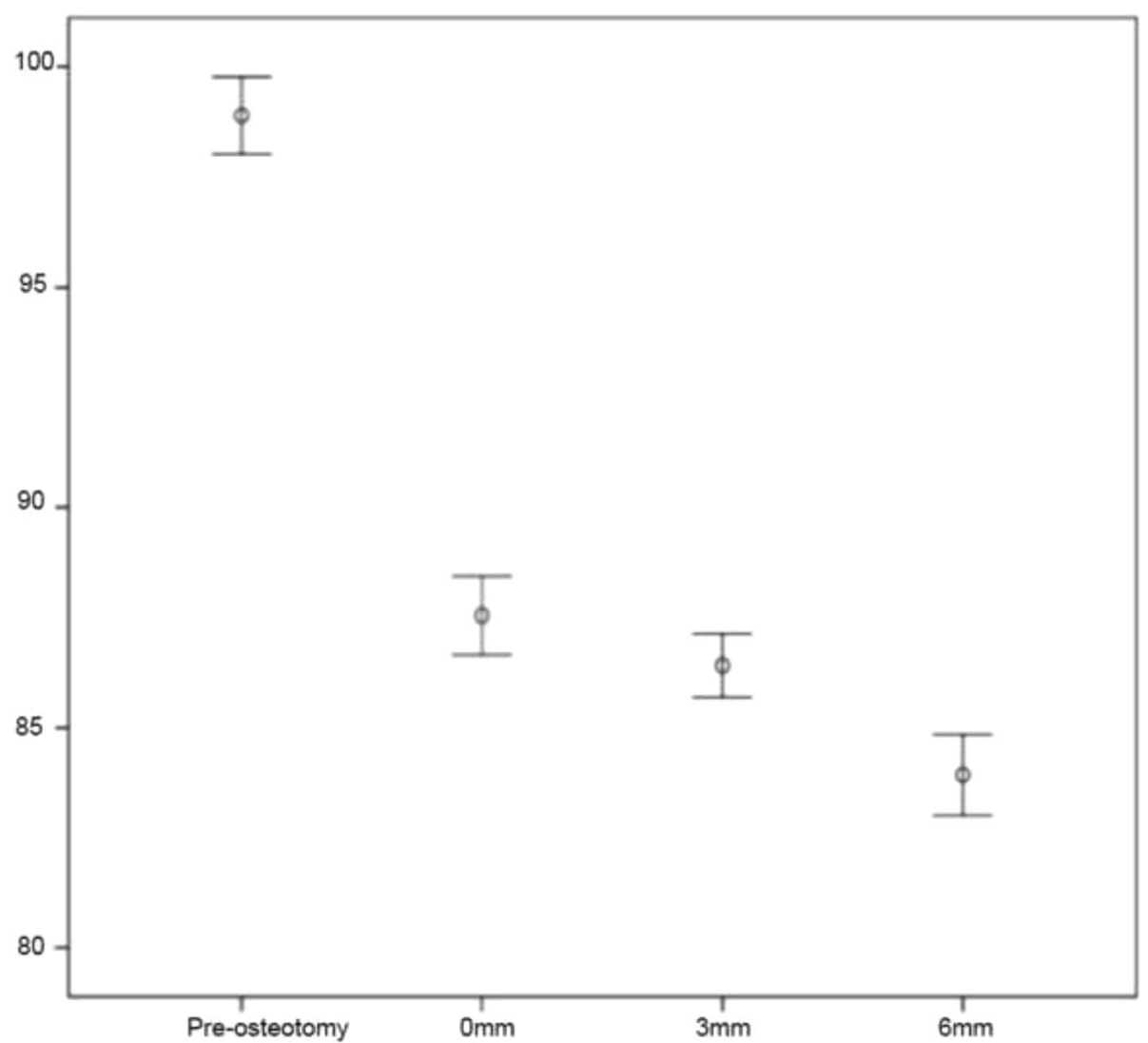

Fig. 2 Patellar Ligament Angle, as measured by the common tangent technique. Error chart (+/- 1 SE) representing the change in PLAtangent $\left({ }^{\circ}\right)$ between tibial tuberosity proximal translation stages. Statistically significant difference was observed between the $0 \mathrm{~mm}$ and $6 \mathrm{~mm}$ stage and $3 \mathrm{~mm}$ and $6 \mathrm{~mm}$ stage

The exact distance of proximal translation necessary to maintain patellar position in clinical cases is unknown and does depend on size of the animal, length of the osteotomy, shape of the proximal tibia, and cage size used. In this study, if the tibial tuberosity was proximalized by $6 \mathrm{~mm}$ the patella was still displaced distally as compared to pre-osteotomy, although this difference was no longer statistically significant. Patella alta was therefore not a result in any of our specimens. The $3 \mathrm{~mm}$ and $6 \mathrm{~mm}$ tibial tuberosity proximalization distances were selected so as to mimic the author's (KV) clinical experience with the described TTA technique $[8,10]$.

Clinically, it seems that in some cases there is a discrepancy between preoperative measurements and postoperative outcome in that the postoperative PLAs tend to be larger than expected, resulting in inadequate advancement of the tibial tuberosity and insufficient dynamic stability of the stifle joint. One study based on a MMP type procedure found that with larger cage sizes and steeper tibial plateau slopes, a larger discrepancy between preoperative measurements and postoperative outcome can be expected [20]. This seems to occur because the direction of the preoperative measurements along the axis of the tibial plateau or common tangent differs to the direction of advancement achieved clinically when the tibial tuberosity is rotated around its distal attachment. Clinically, the direction of advancement is more distal than the direction calculated on preoperative measurements, creating a discrepancy in the distance required to sufficiently advance the tibial tuberosity to the preoperative plan. When proximalizing the tibial tuberosity during the advancement, the tibial tuberosity also shifts more cranially which somewhat offsets the loss of advancement. This effect was significant in the current study between the $0 \mathrm{~mm}$ and the $6 \mathrm{~mm}$ proximalization stages. The actual change in PLA between the $0 \mathrm{~mm}$ and $6 \mathrm{~mm}$ proximalizations was approximately $2.5^{\circ}$ for the PLAslope and $4^{\circ}$ for the PLAtangent in the cadavers evaluated in this study. Based on the current literature, it is unknown whether a relatively small increase in PLA would be of clinical importance, as good clinical outcomes have been reported even with suboptimal post-operative PLA [31].

While no consistent or significant relationship between PLA at $0 \mathrm{~mm}$ and $3 \mathrm{~mm}$ proximalizations was found, there was a continuous decrease in PLA as the tibial tuberosity was proximalized. The decrease in PLA with increasing proximalization was not linear. There was a smaller 


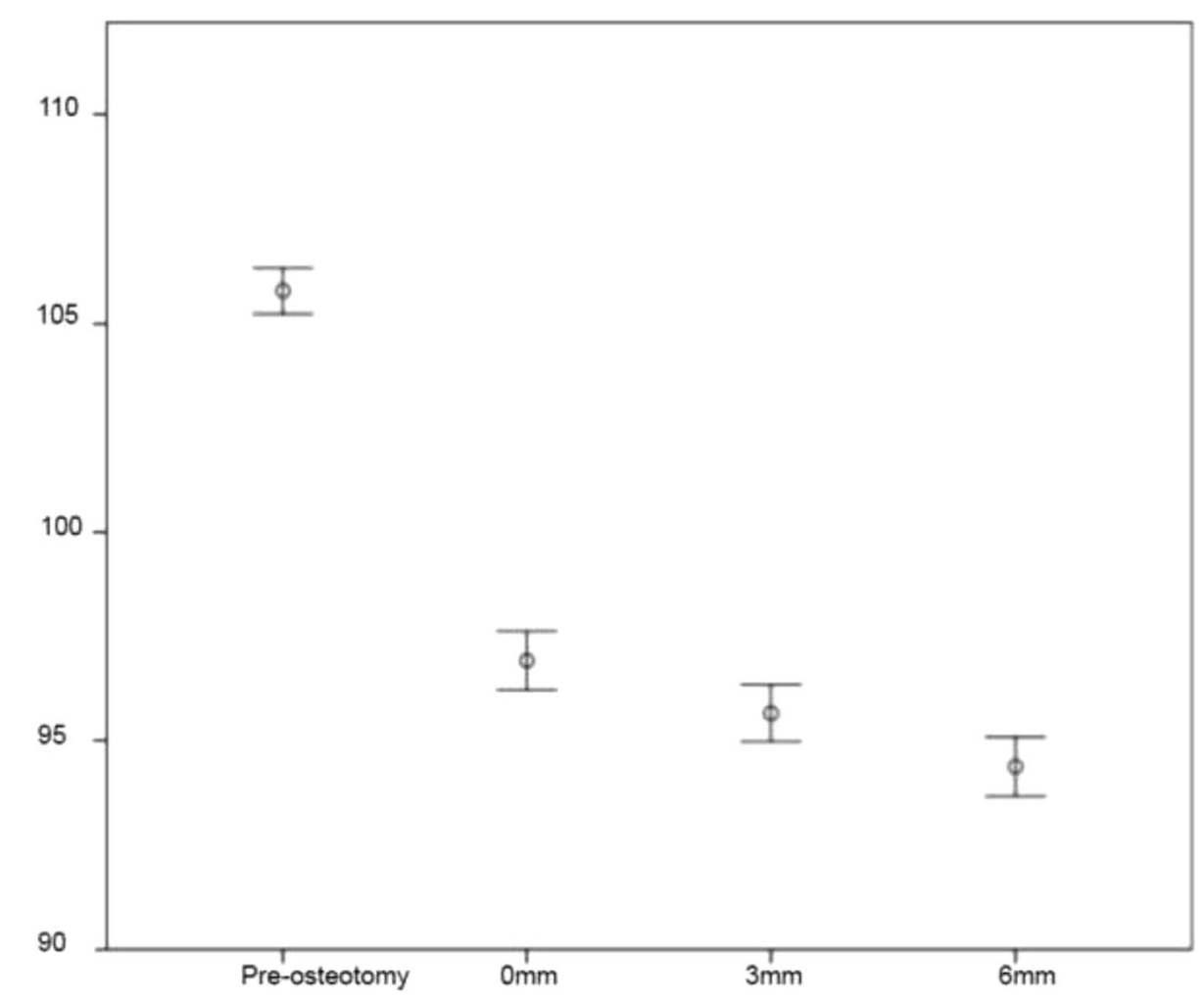

Fig. 3 Patellar Ligament Angle, as measured by the tibial plateau slope technique. Error chart (+/- $1 \mathrm{SE})$ representing the change in PLAslope $\left({ }^{\circ}\right)$ between tibial tuberosity proximal translation stages. Statistically significant difference was observed between the $0 \mathrm{~mm}$ and $6 \mathrm{~mm}$ stage

decrease in PLA between the $0 \mathrm{~mm}$ and $3 \mathrm{~mm}$ stage than between the $3 \mathrm{~mm}$ and $6 \mathrm{~mm}$ stage. This was most likely due to the cage shifting proximal to a larger degree from the $0 \mathrm{~mm}$ to the $3 \mathrm{~mm}$ stage of proximalization than from the $3 \mathrm{~mm}$ to $6 \mathrm{~mm}$ stage in most specimens. Even slight proximal displacement of the cage within the osteotomy wedge will naturally result in reduced advancement of the tibial tuberosity. Whilst we acknowledge that the observed movement of the cages between conditions is a limitation of the accuracy of the study, it should not have influenced the significance of our results; proximal displacement of the cage results in reduction of the advancement, which would have lessened rather than increased the effect of proximalization of the tuberosity. Tilting of the cage ears and subsequent proximal dislodgement of the cage could likely have been avoided by tilting the cage ears prior to fixing the caudal cage ear to the tibial metaphysis.

The same pattern was observed for the PLAslope and PLAtangent methods. There is currently no definitive study in the veterinary literature to determine whether we should aim for a $90^{\circ}$ PLAtangent or PLAslope value when determining TTA cage size preoperatively. It has been suggested that the common tangent method represents a more functionally accurate measurement than the tibial slope method [32] however conversely, the common tangent method has also been found to represent a lesser degree of advancement [33]. In this study, using a compromise of cage size between the two methods, it was expected that there was an overall apparent overcorrection when using post-osteotomy common tangent measurements, and an undercorrection when using the tibial plateau slope measurements. The difference between each of the measurements was as high as $11^{\circ}$ within one specimen in this study. A recent study demonstrated that $70 \%$ of dogs that had undergone a TTA and had a mean postoperative PLAtangent of $89^{\circ}$ continue to have cranial tibial subluxation when weight-bearing, suggesting that further in vivo studies need to be performed to determine which of the measurements would result in optimal clinical outcomes [34]. Certainly, our results suggest that if an MMP type procedure is performed using the same pre-operative planning techniques [33] to those recommended for the original TTA procedure $[8,10]$, this may result in underadvancement of the tibial tuberosity.

There were several limitations of the study that must be acknowledged. Although the blinding method used did not allow assessment of the degree of proximalization of the tibial tuberosity itself, it became apparent during the study that in the proximalized stages the cage ear insert of the cage tended to tilt, and to some observers it was apparent that these radiographs represented specimens in which there was a proximalization of the tibial tuberosity. 
It was not apparent though whether the proximalization was $3 \mathrm{~mm}$ or $6 \mathrm{~mm}$ as the tilting of the cage ear occurred between completing the osteotomy and proximalization of the tibial tuberosity. Another limitation is that the tension of the rubber band tensioning device in the patellar ligament was not standardised or measured, however there was a narrow distance range $(34-43 \mathrm{~mm})$ between the end of the suture loops and pin with a standard four passes between these anchoring points, so that the tension exerted by the rubber band would have been similar between specimens. The procedures and radiographs were carried out in one session per specimen and it is unlikely that stretching of the rubber band would have resulted in decreasing tension during the experiment. Whilst the small sample size of this study is a potential limitation, the excellent interobserver agreement between measurements in all groups as well as statistically significant differences demonstrated between different tibial tuberosity positions show that our results can be interpreted as an indication of the clinical result of the TTA on patellar position and patellar ligament angle. The small sample size of six limbs was selected given the close standardization of the methods and similar conformation of the limbs, minimizing confounding variables.

In our study, variables that could affect the degree of advancement of the tibial tuberosity with a given cage size included the length of the osteotomy, tibia plateau slope, shape of the tibial tuberosity and position of the cage in relation to the level of tibial tuberosity. Whilst we were not able to control these variables in our study, similarly to the clinical situation, our statistical analysis meant that each specimen was compared to itself and therefore interference of these variables with the results was avoided. Patellar position measurements may have also been affected by a number of factors in our study. Positioning the limbs without mimicking weight-bearing, as well as the removal of surrounding soft tissues may have changed the patella position compared to an in vivo scenario however the standardization of this methodology made the results comparable.

The results reported in this study have been obtained with large breed specimens and as such, the magnitudes of patella position and patella ligament angle measurements may not be directly comparable in smaller breed dogs or dogs with different tibial conformations. Similarly, the use of solely left pelvic limbs in our study meant that comparison between right and left was not possible, and therefore symmetry in our results could not be assessed.

\section{Conclusions}

In this report, we show that proximalization of the osteotomized tibial tuberosity during the TTA procedure in a cadaveric model is necessary to maintain vertical patellar position. Proximalization of the tibial tuberosity also reduces post-osteotomy PLA as compared to no proximalization and is another factor that should be considered during preoperative planning.

\section{Methods \\ Cadaver preparation}

Left hind limbs of six large-breed dogs were included in the study. The dogs had been euthanized for reasons unrelated to the study and subsequently stored at $-20{ }^{\circ} \mathrm{C}$. Use of the cadavers was in accordance with the guidelines of the animal ethics committee of the institution. The left hind limbs were disarticulated at the coxofemoral joint once the cadavers were thawed at room temperature. The limbs were prepared by removing skin between the tarsal and coxofemoral joints together with the biceps femoris, hamstrings, and gastrocnemius muscles. The quadriceps muscle was transected approximately $2 \mathrm{~cm}$ proximal to the insertion on the patella and removed proximally. The joint capsule, collateral ligaments, patella and patellar ligament were left intact.

Two $3.2 \mathrm{~mm}$ end-threaded half pins were placed centrally in the diaphysis of the tibia and the femur in a caudocranial direction to create anchor points for mounting the limb to a polymethylmethacrylate (Plexiglass) base caudally. A third pin was placed into the distal third of the femur, also in a caudocranial direction; the pin protruded cranially to create an anchor point for the patella proximally. The limbs were mounted to the Plexiglass sheet using additional pins and small external fixator clamps ${ }^{1}$ with the medial side of the limb facing away from the sheet (Fig. 4). Stifle joint angle was measured with a goniometer, using the following landmarks; greater trochanter of the femur, midpoint between the fibular head and the centre of the lateral femoral condyle, and the lateral malleolus $[35,36]$. The pins and clamps were adjusted until the limb was positioned parallel to the Plexiglass base with the stifle joint at an angle of $135^{\circ}$. The patella was attached to the cranially protruding femoral pin by creating a double loop of 3.5 metric polydioxanone (PDS) in the remaining insertion of the quadriceps muscle at the proximal patella and passing a rubber band ${ }^{2}$ between the suture loop and the pin four times. The tension created by the rubber loop was subjectively assessed as being sufficient to prevent any laxity in the patellar ligament. The length of the 4-fold rubber loop was between $34-43 \mathrm{~mm}$ in all specimens to minimize variability in the tension applied to the patellar ligament.

\section{Pre-osteotomy cage selection}

A mediolateral pre-osteotomy radiograph was obtained to assess femoral and tibial position. Radiographs were obtained at $65 \mathrm{kVp}, 160 \mathrm{~mA}$ and $100 \mathrm{~ms}$ at a distance of $1 \mathrm{~m}$ with a generator. ${ }^{3}$ A computed radiography cassette ${ }^{4}$ was processed in a computed radiography reader. ${ }^{5}$ The positioning was readjusted until the limbs were deemed 


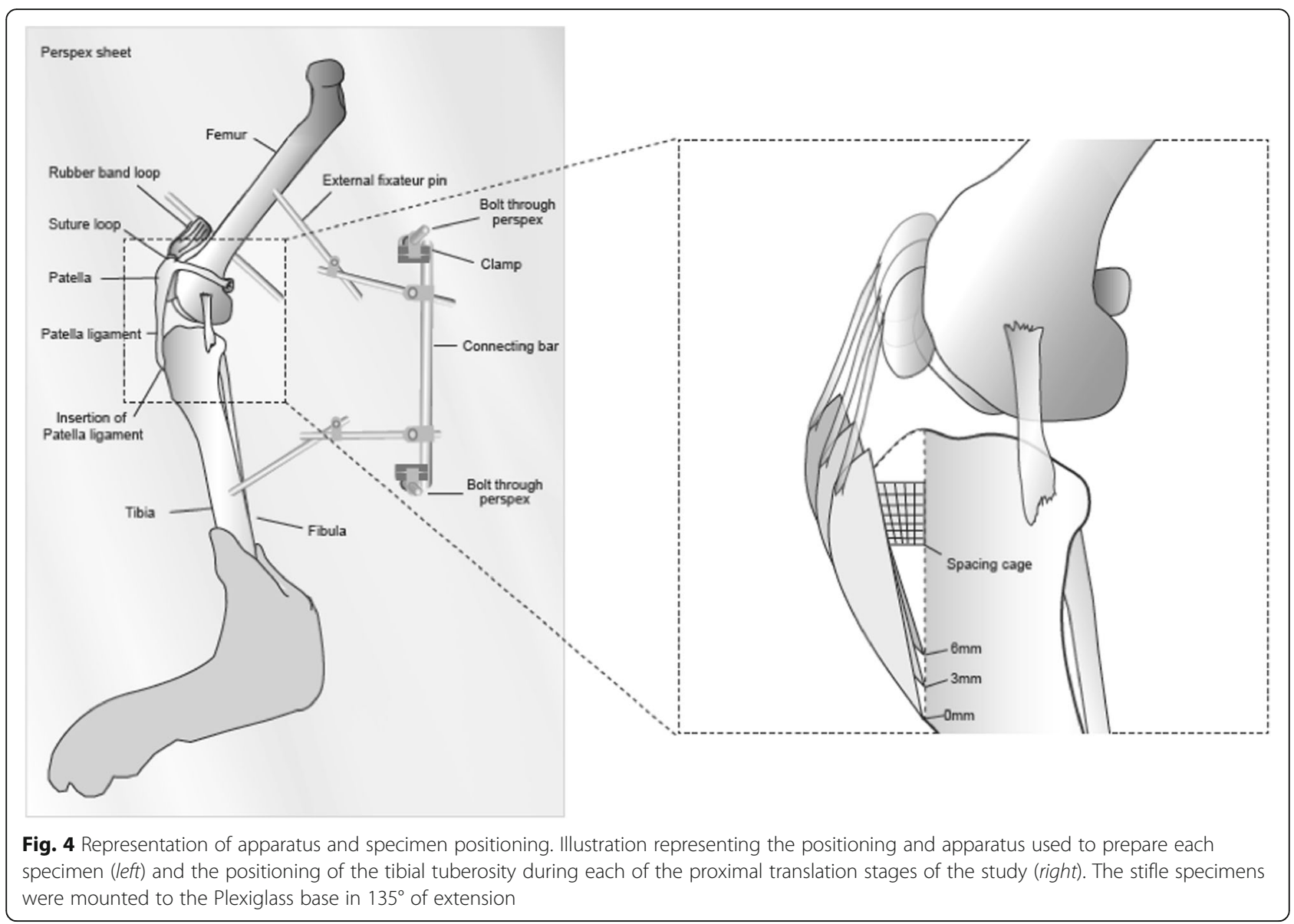

to have minimal femoral or tibial rotation. The required cage size was determined using both the tibial plateau and the common tangent method [21]. If the two measurements indicated cage sizes that differed by two sizes based on manufacturer recommendations ${ }^{6}$, a cage of intermediate size was used (for example a $10.5 \mathrm{~mm}$ cage was used if the common tangent method indicated a $9 \mathrm{~mm}$ cage and tibial plateau slope indicated a $12 \mathrm{~mm}$ cage). If the required cage sizes from each of the measurements were one size apart, with no intermediate size between the measurements, the larger cage was used.

\section{Osteotomy procedure}

A tibial tuberosity osteotomy was performed perpendicular to the sagittal plane of the tibia from a point cranial to the medial meniscus to the distal aspect of the tibial tuberosity using an oscillating saw. Initially, the osteotomy was incomplete distally [11], leaving a cortical hinge of approximately $2-3 \mathrm{~mm}$ thickness. The tibial tuberosity was advanced without breaking the distal cortical hinge and the pre-selected cage was placed into the osteotomy gap, with the distal edge of the cage placed at the level of the tibial tuberosity. A $0.9 \mathrm{~mm}$ Kirschner wire was inserted into the tibial metaphysis through the caudal cage ear for stabilization of the cage within the osteotomy gap. A second mediolateral radiograph was obtained (0mm radiograph).

The osteotomy was then completed distally, and the tibial tuberosity was shifted proximally by $3 \mathrm{~mm}$. The tibial tuberosity was secured in place with large pointed bone reduction forceps placed perpendicular to the osteotomy plane with the distal end of the tibial tuberosity in contact with the tibial diaphysis. A third mediolateral radiograph was obtained. The same procedure was repeated with the tibial tuberosity translated proximally by $6 \mathrm{~mm}$.

\section{Radiographic assessment}

Radiographs were checked to ensure that there had not been any movement or rotation of the specimen in relation to the external fixation and Plexiglass sheet, which could have changed the position of the specimen from the pre-osteotomy stage. Position of the cage was also assessed after each stage of proximalization. In most specimens the cage tended to shift slightly proximal during proximalization of the tibial tuberosity due to tilting of the caudally secured cage ear insert within the cage. This was deemed to be acceptable providing the cage had not tilted. 


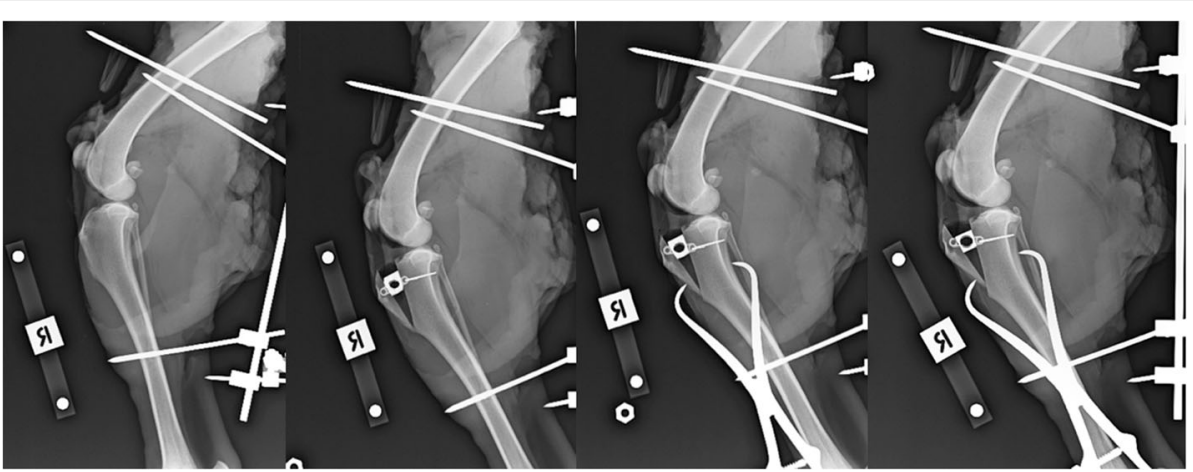

Fig. 5 Series of the four lateral radiographs within one specimen prior to blinding. From left to right: Pre-osteotomy, and 0mm, 3mm, and $6 \mathrm{~mm}$ proximalization of the tibial tuberosity

Once the series of pre-osteotomy, $0 \mathrm{~mm}, 3 \mathrm{~mm}$ and $6 \mathrm{~mm}$ radiographs were obtained for each of the 6 specimens (Fig. 5), the radiographs were blinded. This was performed using a blinding method in which the 24 radiographs to be assessed were selected on $\mathrm{eFilm}^{7}$, exported to Osirix ${ }^{8}$, and cropped such that the distal aspect of the osteotomy including the absence or presence of pointed bone reduction forceps was no longer visible (Fig. 3). A random number generator ${ }^{9}$ was used to create a random sequence of all radiographs. Each radiograph was labelled with a number and all radiographs placed on a blank compact disc. This was presented to 3 observers; a specialist veterinary surgeon, a specialist veterinary radiologist, and a veterinary student for assessment of patellar position and patellar ligament angle.

A second set of all non-blinded images was randomised in the same way as described. The radiographs were calibrated and one observer (veterinary student) measured radiographic tibial tuberosity proximalization distance in the $3 \mathrm{~mm}$ and $6 \mathrm{~mm}$ stage radiographs with the measurement tool of the Osirix software ${ }^{h}$. The distance between the distal ends of the osteotomized edges of the tibial tuberosity and tibial metaphysis was measured on two separate occasions and averaged. This average was used to determine mean (+/-SD) tibial tuberosity proximalization distance for each stage.

\section{Patellar position measurement}

Patellar position was measured using a modification of several indirect methods, mostly resembling the Blackburne-Peel method [26-28, 37]. Patellar articular length was measured from the base to the apex. A perpendicular measurement from the common tangent of the femoro-tibial articulation to the midpoint of the patellar articular surface was recorded (Fig. 6) and this measurement was used as the PP value.

\section{Patellar ligament angle measurement}

Patellar ligament angle was measured using both tibial plateau slope (PLAslope) and the common tangent (PLAtangent) as a reference line to the patellar ligament. The tibial plateau slope was defined by the cranial and caudal extent of the medial tibial condyle [38]. The common tangent was evaluated by creating a line perpendicular to a line connecting the centres of superimposing circles corresponding to the respective articular surfaces of the femoral condyles and the medial tibial condyle.

\section{Statistical analysis}

Statistical analysis was performed using a statistical software package. ${ }^{10}$ Data are presented as mean \pm standard deviation (SD). Measurements from the three observers were pooled into pre-osteotomy,

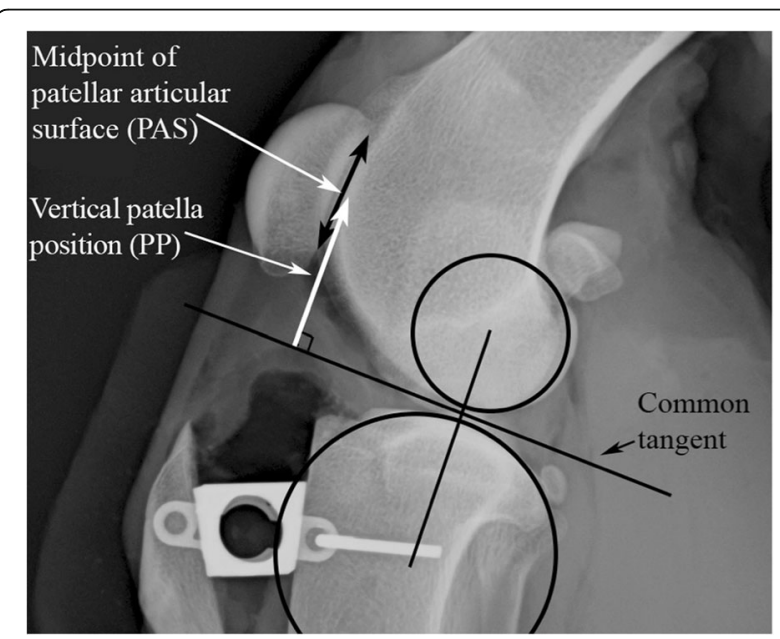

Fig. 6 Vertical patellar position measurement. Patellar position was measured as the distance $(\mathrm{mm})$ from the midpoint of the patellar articular surface perpendicular to the common tangent of femoral and tibial condyles. Note that this radiograph is blinded in that the degree of proximalization is not visible 
0mm, $3 \mathrm{~mm}$ and $6 \mathrm{~mm}$ covariants for PP, PLAslope and PLAtangent. Shapiro-Wilk test was used to confirm normal distribution of the pooled data within each of the measurement stages (pre-osteotomy, $0 \mathrm{~mm}, 3 \mathrm{~mm}$ and $6 \mathrm{~mm}$ ) for PP, PLAtangent and PLAslope. The assumption of sphericity was confirmed for each of these stages using Mauchly's Test of Sphericity. Repeated measures ANOVA was used to test for differences between pre-osteotomy PP and PP at the different proximalization stages, and to compare PLAslope and PLAtangent between the different proximalization stages. Pair-wise comparisons were used for PP, PLAslope and PLAtangent covariant measurement groups where results of the ANOVA were significant $(\mathrm{P}<0.05)$ and post-hoc Bonferroni adjustments allowed for multiple comparisons within each of these categories.

Additionally, interobserver agreement was calculated based on intraclass correlation coefficient (ICC) analysis, measuring absolute agreement, where 0 represented no agreement/consistency and 1 represented perfect agreement/consistency. Excellent agreement was defined as ICC $>0.8$.

\section{Endnotes}

${ }^{1}$ Small EK ESF Clamp, Imexx Veterinary Inc., Longview, Texas

${ }^{2}$ Size 64 rubber band, Esselte Australia Pty Ltd, Sydney, Australia

${ }^{3}$ Toshiba KXO-60G generator, Toshiba Asia-Pacific, Singapore, Singapore

${ }^{4} 35 \times 43 \mathrm{~cm}$ FCR Fuji IP, Type CC Cassette, Fujifilm, Tokyo, Japan

${ }^{5}$ FCR Profect One Reader, Fujifilm, Tokyo, Japan

${ }^{6}$ TTA preoperative planning guide, Kyon, Zurich, Switzerland

${ }^{7}$ eFilm System 3.4, Merge Healthware, Chicago, Ilinois ${ }^{8} 32$-bit DICOM viewer, Osirix, Geneva, Switzerland

${ }^{9}$ Research Randomizer, Version 3.0, Retrieved on April

22, 2011, from http://www.randomizer.org/

${ }^{10}$ SPSS20.0, IBM, New York, NY

\section{Abbreviations}

CrCL: Cranial cruciate ligament; MMP: Modified Maquet procedure; PLA: Patellar ligament angle; PLAslope: Patellar ligament angle derived from the tibial plateau slope; PLAtangent: Patellar ligament angle derived from the common tangent; PP: Patellar position; TTA: Tibial tuberosity advancement

\section{Acknowledgements}

Not applicable.

\section{Funding}

No funding was obtained for this research.

\section{Availability of data and materials}

The datasets during and/or analysed during the current study available from the corresponding author on reasonable request.

\section{Authors' contributions}

JNT and KV were involved in preparation of specimens, radiograph analysis, analysis and interpretation of data and manuscript preparation. MM contributed to analysis of radiographs and data as well as data interpretation. $\mathrm{KJ}$ contributed to the experimental design, interpretation of the data and revision of the manuscript for intellectual content. All authors read and approved the final manuscript.

\section{Competing interests}

The authors declare that they have no competing interests.

\section{Consent for publication}

Not applicable.

\section{Ethics approval and consent to participate}

This research was conducted in accordance with institutional animal ethics policies.

Received: 29 August 2015 Accepted: 4 January 2017

Published online: 09 January 2017

\section{References}

1. Johnson JA, Austin C, Breur GJ. Incidence of canine appendicular musculoskeletal disorders in 16 veterinary teaching hospitals from 1980 to 1989. Vet Comp Orthop Traumatol. 1994;7:56-9.

2. Innes JF, Bacon D, Lynch C, Pollard A. Long-term outcome of surgery for dogs with cranial cruciate ligament deficiency. Vet Rec. 2000;147:325-8.

3. Elkins AD, Pechman R, Kearney MT, Herron M. A retrospective study evaluating the degree of degenerative joint disease in the stifle joints of dogs following surgical repair of anterior cruciate ligament. J Am Anim Hosp Assoc. 1991;27:533-40.

4. Witsberger TH, Villamil JA, Schultz LG, Hahn AW, Cook JL. Prevalence of and risk factors for hip dysplasia and cranial cruciate ligament deficiency in dogs. J Am Vet Med Assoc. 2008;232:1818-24.

5. Etchepareborde S, Barthelemy N, Mills J, Pascon F, Ragetly GR, Balligand M. Mechanical testing of a modified stabilisation method for tibial tuberosity advancement. Vet Comp Orthop Traumatol. 2010;23:400-5.

6. Slocum B, Devine T. Cranial tibial wedge osteotomy: a technique for eliminating cranial tibial thrust in cranial cruciate ligament repair. J Am Vet Med Assoc. 1984;184:564-9.

7. Slocum B, Slocum TD. Tibial plateau levelling osteotomy for repair of cranial cruciate ligament rupture in the canine. Vet Clin North Am Small Anim Pract. 1993;23:777-95.

8. Montavon PM, Damur D, Tepic S. Advancement of the tibial tuberosity for the treatment of the cranial cruciate ligament deficient canine stifle, Proceedings, $1^{\text {st }}$ World Orthopaedic Veterinary Congress. ESVOT-VOS, Munich, 2002

9. Bruce WJ, Rose A, Tuke J, Robins GM. Evaluation of the Triple Tibial Osteotomy. A new technique for the management of the canine cruciate-deficiency stifle. Vet Comp Orthop Traumatol. 2007;20:159-68.

10. Lafaver S, Miller N, Stubbs W, Taylor R, Boudrieau T. Tibial tuberosity advancement for stabilization of the cranial cruciate ligament-deficient stifle joint: Surgical technique, early results, and complications in $101 \mathrm{dogs}$. Vet Surg. 2007;36:573-86.

11. Etchepareborde S, Brunel L, Bollen G, Balligand M. Preliminary experience of a modified technique for repair of cranial cruciate ligament rupture in dogs. Vet Comp Orthop Traumatol. 2011;24:223-7.

12. Ness M. The Modified Maquet Procedure (MMP) in Dogs: Technical Development and Initial Clinical Experience. J Am Anim Hosp Assoc. 2016;52:242-50.

13. Maquet P. Advancement of the tibial tuberosity. Clin Orthop Relat Res. 1976; 115:225-30.

14. Kapler M, Marcellin-Little DJ, Roe SC. Planned wedge size compared to achieved advancement in dogs undergoing the modified Maquet procedure. Vet Comp Orthop Traumatol. 2015;28:379-84.

15. Samoy Y, Verhoeven G, Bosmans T, Van der Vekens E, de Bakker E, Verleyen P. Van RyssenB. TTA Rapid: Description of the Technique and Short Term Clinical Trial Results of the First 50 Cases. Vet Surg. 2015;44:474-84.

16. Brunel L, Etchepareborde S, Barthelemy N, Farnier F, Balligand M. Mechanical testing of a new osteotomy design for tibial tuberosity advancement using the Modified Maquet Technique. Vet Comp Orthop Traumatol. 2013;26:47-53. 
17. Edwards GA, Jackson AH. Use of a TTA Plate for the Correction of Severe Patella Baja in a Chihuahua. J Am Anim Hosp Assoc. 2012;48:113-7.

18. Backstein D, Meisami B, Gross AE. Patella Baja After the Modified CoventryMaquet High Tibial Osteotomy. J Knee Surg. 2003;16:203-8.

19. Insall J, Salvati E. Patella position in the normal knee joint. Radiology. 1971; 101:101-4.

20. Etchepareborde S, Mills J, Busoni V, Brunel L, Balligand M. Theoretical discrepancy between cage size and efficient tibial tuberosity advancement in dogs treated for cranial cruciate ligament rupture. Vet Comp Orthop Traumatol. 2011;24:27-31.

21. Millet M, Bismuth C, Labrunie A, Marin B, Filleur A, Pillard P, et al. Measurement of the patellar tendon-tibial plateau angle and tuberosity advancement in dogs with cranial cruciate ligament rupture; reliability of the common tangent and tibial plateau methods of measurement. Vet Comp Orthop Traumatol. 2013:26:469-78.

22. Schwandt CS, Bohorquez-Vanelli A, Tepic S, Hassig M, Dennler R, Vezzoni A, et al. Angle between the patellar ligament and the tibial plateau in dogs with partial rupture of the cranial cruciate ligament. Am J Vet Res. 2006;67:1855-60.

23. Reif U, Dejardin LM, Probst CW, DeCamp CE, Flo GL, Johnson AL. Influence of limb positioning and measurement method on the magnitude of the tibial plateau angle. Vet Surg. 2004;33:368-75.

24. Bielecki M, Schwandt CS, Scharvogel S. Effect of tibial subluxation on the measurements for tibial tuberosity advancement in dogs with cranial cruciate ligament deficiency. Vet Comp Orthop Traumatol. 2014;27:470-7.

25. Bismuth C, Ferrand FX, Millet M, Marin B, Filleur A, Pillard P, et al. Comparison of radiographic measurements of the patellar tendon-tibial plateau angle with anatomical measurements in dogs. Vet Comp Orthop Traumatol. 2014;27:222-9.

26. Blackburne JS, Peel T. A new method of measuring patella height. J Bone Joint Surg. 1977;59-B:241-2

27. Rogers BA, Thornton-Bott P, Cannon SR, Briggs TW. Interobserver variation in the measurement of patella height after total knee arthroplasty. J Bone Joint Surg. 2006;88-B:484-8.

28. Miles JE, Nielsen DH, Jensen BR, Kirpensteijn J, Svalastoga EL, Eriksen T. Comparison of reliability of five patella position indices at various stifle joint angles in pelvic limbs obtained from cadavers of red foxes (Vulpes vulpes). Am J Vet Res. 2012;73:263-71.

29. Egund N, Lundin A, Wallengren NO. The vertical position of the patella: a new radiographic method for routine use. Acta Radiol. 1988;29:555-8.

30. Mostafa A, Griffon D, Thomas M, Constable PD. Proximodistal alignment of the canine patella: radiographic evaluation and association with medial and lateral patella luxation. Vet Surg. 2011;32:201-11.

31. Hoffmann DE, Miller JM, Ober CP, Lanz OI, Martin RA, Shires PK. Tibial tuberosity advancement in 65 canine stifles. Vet Comp Orthop Traumatol. 2006;19:219-27.

32. Hoffmann D, Kowaleski MP, Johnson KA, Evans RB, Boudrieau RJ. Ex Vivo biomechanical evaluation of the canine cranial cruciate ligament-deficient stifle with varying angles of stifle joint flexion and axisal loads after tibial tuberosity advancement. Vet Surg. 2011;40:311-20.

33. Cadmus J, Palmer RH, Duncan C. The effect of preoperative planning method on recommended tibial tuberosity advancement cage size. Vet Surg. 2014:43:995-1000.

34. Skinner OT, Kim SE, Lewis DD, Pozzi A. In vivo femorotibial subluxation during weight-bearing and clinical outcome following tibial tuberosity advancement for cranial cruciate ligament insufficiency in dogs. Vet J. 2013; 196:86-91.

35. DeCamp CE, Soutas-Little RW, Hauptman J, Olivier B, Braden T, Walton A Kinematic gait analysis of the trot in healthy greyhounds. Am J Vet Res. 1993;54:627-34

36. Bush MA, Bowlt K, Gines J, Owen MR. Effect of use of different landmark methods on determining stifle angle and on calculated tibial tuberosity advancement. Vet Comp Orthop Traumatol. 2011;24:205-10.

37. Phillips CL, Silver DAT, Schranz PJ, Mandalia V. The measurement of patella height; a review of the methods of imaging. J Bone Joint Surg. 2010;92-B: 1045-53.

38. Baroni E, Matthias R, Marcellin-Little D, Vezzoni A, Stebbins ME. Comparison of radiographic assessments of the tibial plateau slope in dogs. Am J Vet Res. 2006;64:586-9.

\section{Submit your next manuscript to BioMed Central and we will help you at every step:}

- We accept pre-submission inquiries

- Our selector tool helps you to find the most relevant journal

- We provide round the clock customer support

- Convenient online submission

- Thorough peer review

- Inclusion in PubMed and all major indexing services

- Maximum visibility for your research

Submit your manuscript at www.biomedcentral.com/submit
Biomed Central 